\title{
Oxidative stress is important in the pathogenesis of stress-related mucosal disease
}

\author{
XIAOHONG WANG ${ }^{1,2}$, QIN ZHAO $^{1,2}$, HUANLING SHI $^{3}$, FENG $^{4}, \mathrm{NA} \mathrm{SHI}^{5}$, \\ DONGFANG BAI ${ }^{6}$, XIAOPEI $\mathrm{LI}^{2}$, HAIPENG YUAN ${ }^{2}$ and XIULI ZUO ${ }^{1}$ \\ ${ }^{1}$ Department of Gastroenterology, Qilu Hospital of Shandong University, Jinan, Shandong 250012; \\ Departments of ${ }^{2}$ Gastroenterology, ${ }^{3}$ Endoscopy Center, ${ }^{4}$ Critical Care Medicine, \\ ${ }^{5}$ Central Laboratory and ${ }^{6}$ Endocrinology, Tai'an Central Hospital, Tai'an, Shandong 271000, P.R. China
}

Received March 8, 2019; Accepted June 24, 2020

DOI: $10.3892 / \mathrm{etm} .2020 .9211$

\begin{abstract}
Stress-related mucosal disease (SRMD) is a common complication in patients in the intensive care unit (ICU). The aim of the present study was to investigate the possible mechanisms for the pathogenesis of SRMD. In total, 38 patients with SRMD were enrolled from an ICU, as well as 15 healthy volunteers. The disease severity of patients in ICU was evaluated using the Acute Physiology and Chronic Health Evaluation (APACHE) II score. Gastric mucosa with the most severe lesions were biopsied for hematoxylin and eosin staining and then assessed by pathological damage scoring. The serum levels of malondialdehyde (MDA), superoxide dismutase (SOD) and ischemic modified albumin (IMA) were also detected. In addition, claudin-3 and inducible nitric oxide (NO) synthase (iNOS) in the gastric mucosa were assessed by western blotting and immunohistochemistry. The average APACHE II score of the patients with SRMD was significantly higher compared with the controls. Moreover, the levels of MDA $(4.74 \pm 2.89 \mathrm{nmol} / \mathrm{ml})$ and IMA $(93.61 \pm 10.78 \mathrm{U} / \mathrm{ml})$ in patients with SRMD were significantly higher compared with the controls $(\mathrm{P}<0.001)$, while those of SOD $(89.66 \pm 12.85 \mathrm{U} / \mathrm{ml})$ in the patients with SRMD were significantly lower compared with the controls $(\mathrm{P}<0.001)$. Furthermore, compared with the control, iNOS expression was significantly higher $(\mathrm{P}=0.034)$, while the expression of claudin-3 was significantly lower in patients with SRMD $(\mathrm{P}<0.001)$. The results indicated that APACHE II score was positively correlated with pathological damage score $(\mathrm{r}=0.639, \mathrm{P}<0.001)$ and levels of MDA $(\mathrm{r}=0.743, \mathrm{P}<0.001)$, but negatively correlated with the level of SOD $(r=-0.392, P=0.015)$. In addition, MDA was positively
\end{abstract}

Correspondence to: Professor Xiuli Zuo, Department of Gastroenterology, Qilu Hospital of Shandong University, 107 Wenhuaxi Road, Jinan, Shandong 250012, P.R. China

E-mail: zuoxiuli@sdu.edu.cn

Key words: stress-related mucosal disease, Acute Physiology and Chronic Health Evaluation II score, oxidative stress, malondialdehyde, inducible NO synthase correlated with IMA $(\mathrm{r}=0.380, \mathrm{P}=0.018)$, but negatively correlated with claudin-3 ( $\mathrm{r}=-0.377, \mathrm{P}=0.020)$. Therefore, it was speculated that oxidative stress may play an important role in the pathogenesis of SRMD, and NO levels and cell membrane permeability are altered during this process.

\section{Introduction}

Stress-related mucosal disease (SRMD) is a common complication in patients of the intensive care unit (ICU) (1). Moreover, endoscopic examination within $72 \mathrm{~h}$ of ICU admission shows that $75-100 \%$ of patients who are critically ill display lesions and 1-6\% patients in ICU have bleeding in the upper gastrointestinal mucosa (2). The pathological stage of SRMD is related to disease severity of patients in ICU (3), thus, the more severe the condition, the higher the pathological damage score. Furthermore, disease severity of patients in ICU is evaluated using the Acute Physiolog $\mathrm{y}$ and Chronic Health Evaluation (APACHE) II score (4). As one of the primary applied scoring systems in ICU, the APACHE II score is widely used in the assessment of various critical illnesses, including disease severity, treatment and prognosis (5).

Oxidative stress, ischemia-reperfusion injury, endogenous nitric oxide (NO) and reduced mucosal blood flow are causative factors of SRMD (6). However, the underlying mechanisms of SRMD are not fully understood. Oxidative stress is the state when there is an imbalance between reactive oxygen species (ROS) and the activity and availability of antioxidants (7). Moreover, assessment of oxidative stress can be achieved mainly via the following two parameters: i) level of malondialdehyde (MDA), which is used as an indicator of lipid peroxidation (8); and ii) activity of superoxide dismutase (SOD), which reflects antioxidant capacity (9). The first stage of ROS-mediated cell damage is peroxidation of the cell membrane matrix; in particular, lipid peroxidation $(9,10)$. In the lipid peroxidation process, a polar group is inserted into the lipid molecule and is settled in the lipid bilayer (9). Therefore, the cell membrane becomes hydrophobic and permeable (9). Furthermore, the products of lipid peroxidation, such as MDA, react with the amines of the cell membrane proteins to produce a Schiff base, so that the cell membrane becomes 
stiffer $(9,11)$. Therefore, the level of MDA is used to evaluate ROS-induced damage in various organs and tissues (11).

Ischemic modified albumin (IMA), which can be induced by oxidative stress, increases within and outside of the heart when ischemia-reperfusion injury occurs (12). Therefore, it is a candidate marker in the assessment of myocardial ischemia, and has also been identified as a marker of oxidative stress $(13,14)$.

NO plays an important role in the regulation of various cellular functions, including those of the gastrointestinal tract (15). In addition, NO is synthesized from L-arginine via NO synthase (NOS), including constitutive NOS (cNOS) and inducible NOS (iNOS) (16). cNOS, includes neuronal NOS and endothelial NOS, which produce small amounts of NO that act as a neurotransmitter and vasodilator, respectively (16). However, iNOS produces larger amounts of NO and is only expressed during inflammation (17). It has been previously reported that NO plays biphasic roles in the ulcerogenic response of the gastric mucosa $(18,19)$. Previous studies have also shown that ischemia-reperfusion injury is often accompanied by inflammation, and iNOS can be expressed by inflammatory cells $(16,20,21)$.

The claudin family of tight junction proteins plays an important role in regulating epithelial paracellular permeability (22). In vitro experiments by Hashimoto et al (23) revealed that the expression of claudin-3 in the gastric epithelium is reduced by $\mathrm{H}_{2} \mathrm{O}_{2}$, a type of ROS. However, the relationship between oxidative stress and claudin-3 expression in gastric mucosal cells of patients with SRMD has not been reported.

Although the pathogenesis of SRMD has been examined in animal models and in vitro, to the best of our knowledge there have been no studies conducted in patients. Therefore, in the present study, patients from ICU were enrolled and the roles of oxidative stress, iNOS and claudin-3 in the pathogenesis of SRMD were investigated.

\section{Materials and methods}

Ethical statement. The study was carried out in Central Hospital of Tai'an (Tai'an, China), from January 2015 to May 2017, and was approved by the Institutional Ethics Committee of Tai'an Central Hospital. All subjects provided their informed consent. The study was registered at www.clinicaltrials.gov (registration no. NCT03200158).

Patients and volunteers. In total, 38 patients in ICU with SRMD were enrolled within $72 \mathrm{~h}$ after hospitalization. These SRMD patients included 20 males and 18 females, with an average age of $47.61 \pm 6.28$ years. All patients were diagnosed by gastroscopy and had gastric mucosal lesion under gastroscopy. Patients with gastric cancer, esophageal cancer or history of taking Aspirin were excluded. In total, 15 age and sex matched healthy volunteers ( 8 females and 7 males; mean age, $47.0 \pm 5.58$ years) were recruited as controls.

APACHE II score. The APACHE II score was completed within $24 \mathrm{~h}$ after ICU admission. The APACHE II scoring system includes 12 physiological parameters (with each item $0-4$ points and a total of $0-60$ points), chronic disease health status (2-5 points) and age (0-6 points); the total score is $0-71$ points $(5)$.
Sample collection. Gastric mucosa and blood samples of subjects were collected within $72 \mathrm{~h}$ after hospitalization. Biopsy gastric mucosal tissues of each individual were taken from four different areas of the most prominent erosion area of gastric mucosa during gastroscopy or the normal gastric antrum (for healthy controls). Peripheral venous blood samples $(6 \mathrm{ml})$ were collected from each individual. The serum was collected after centrifugation at $1,000 \mathrm{xg}$ at $4^{\circ} \mathrm{C}$ for $15 \mathrm{~min}$.

Pathological damage score. Gastric mucosal tissues were fixed with $10 \%$ formalin at room temperature for $24 \mathrm{~h}$ and routinely paraffin embedded, sliced at $3-\mu \mathrm{m}$ and stained with hematoxylin and eosin (H\&E; 60 and $90 \mathrm{sec}$, respectively), at room temperature according to routine procedure. The degree of pathological damage was scored according to the Masuda criteria (24) with a slight modification: i) 0 , superficial epithelial cells were intact and arranged well; ii) 1, surface epithelium was damaged and the neutrophils were infiltrated; iii) 2, upper mucosa was congested or edematous; iv) 3, cells were interrupted, the middle or lower layer of the membrane was congested, with edema or bleeding; v) 4, upper mucosal gland structure was disordered or necrotic; and vi) 5, deep necrosis or ulceration. The mean score of five slides was calculated as the pathological damage score of each patient.

Serum MDA, SOD and IMA measurement. Serum MDA was detected using the MDA assay kit (cat. no. A003-1; Nanjing Jiancheng Bioengineering Institute) as previously described (25).

Serum IMA was measured by the ischemia modified albumin detection kit (https://www.reebio.com/products/product-i328. html; Ningbo Ruiyuan Biological Technology Co., Ltd.). The serum was treated with $5.0 \mathrm{mmol} / 1$ cobalt oxide at $37^{\circ} \mathrm{C}$ for 3-5 min, and serum albumin combined with the cobalt ions and the absorbance value at $405 \mathrm{~nm}\left(\mathrm{~A}_{1}\right)$ was measured using automatic biochemical analyzer $\left(\operatorname{Cobas}^{\circledR}\right.$ 8000; Roche Diagnostics). Subsequently, $2.8 \mathrm{mmol} / 1 \mathrm{chromogenic}$ agent (at $37^{\circ} \mathrm{C}$ for $5 \mathrm{~min}$ ) was added to the residual cobalt ions to produce a reddish brown product and the absorbance value at $510 \mathrm{~nm}\left(\mathrm{~A}_{2}\right)$ were measured. The serum IMA content was calculated using the following formula: IMA concentration $(\mathrm{U} / \mathrm{ml})=\left(\mathrm{A}_{2}-\mathrm{A}_{1}\right) / \Delta \mathrm{A}$ calibration $\mathrm{x}$ IMA calibrator concentration.

The activity of SOD was determined using a SOD detection kit [http://www.co-health.com.cn/content/ nr_fy.jsp?code=hyyksw_cpzx_cpml; Co-Health (Beijing) Laboratories Co., Ltd.;]. After incubation with $10 \mathrm{mmol} / 1$ reagent 1 at $37^{\circ} \mathrm{C}$ for $5 \mathrm{~min}$, the absorbance value $\left(\mathrm{A}_{1}\right.$ $405 \mathrm{~nm}$ ) was measured before $20 \mathrm{mmol} / 1$ reagent 2 (at $37^{\circ} \mathrm{C}$ for $5 \mathrm{~min}$ ) was added and the absorbance value $\left(\mathrm{A}_{2} 510 \mathrm{~nm}\right)$ were measured. SOD concentration in the serum sample was calculated according to that in a standard substance, whose SOD concentration was known, with the following formula: SOD concentration $(\mathrm{U} / \mathrm{ml})=\left(\mathrm{A}_{2}-\mathrm{A}_{1}\right) / \Delta \mathrm{A}$ calibration $\mathrm{x}$ SOD calibrator concentration.

Immunohistochemistry. Measurement of iNOS in gastric mucosa was carried out by immunohistochemistry. The biopsy specimens were fixed with $10 \%$ formalin at room temperature for $24 \mathrm{~h}$ and routinely processed in paraffin 

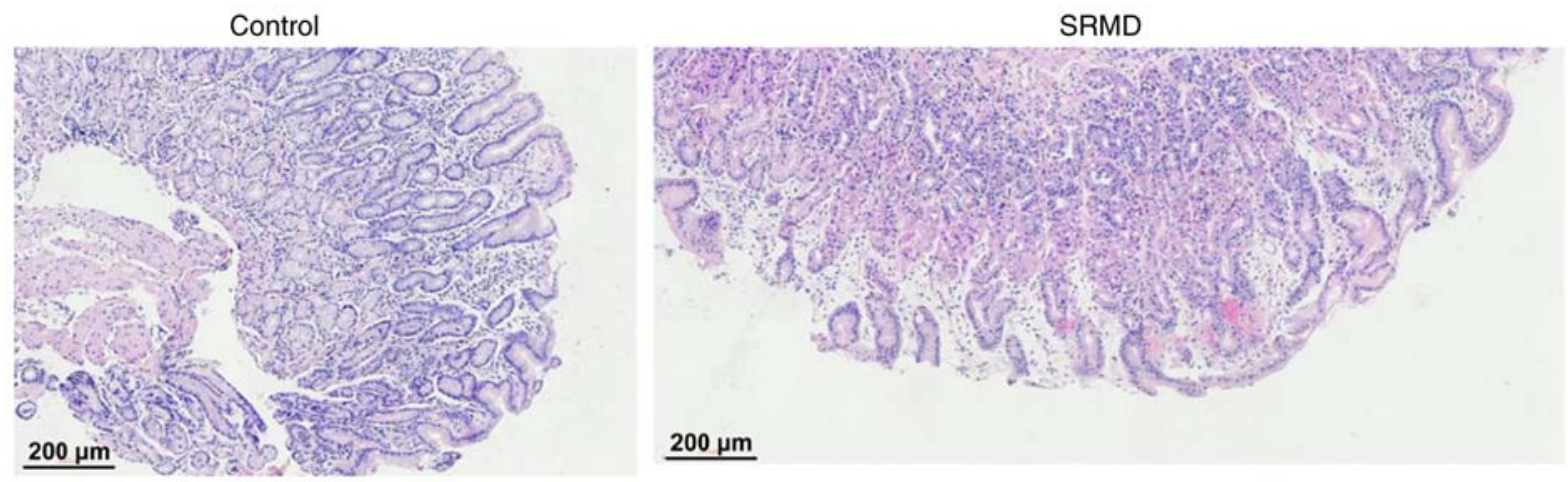

Figure 1. Gastric mucosa from a patient with SRMD and healthy control. Hematoxylin and eosin staining. Magnification x100. Scale bar, $200 \mu \mathrm{m}$. SRMD, stress-related mucosal disease.

and cut into 5- $\mu \mathrm{m}$ sections. The paraffin sections were then dewaxed and washed with PBS, and $50 \mu 10.3 \% \mathrm{H}_{2} \mathrm{O}_{2}$ solution was added and incubated at room temperature for $10 \mathrm{~min}$ to block endogenous peroxidase activity. Subsequently, sections were incubated with $50 \mu 1$ non-immunized animal serum (OriGene Technologies, Inc.) for $10 \mathrm{~min}$ at room temperature before $50 \mu \mathrm{l}$ rabbit anti-human anti-iNOS polyclonal antibody (cat. no. ab53769; 1:200; Abcam) was added and incubated overnight at $4^{\circ} \mathrm{C}$. After washing with PBS, the sections were incubated with $50 \mu \mathrm{l}$ biotin-labeled goat anti-rabbit secondary antibodies (1:1,000; cat. no. TA130016; OriGene Technologies, Inc.) at room temperature for $15 \mathrm{~min}$. After washing again, $50 \mu 1$ peroxidase-labeled streptavidin (cat. no. SP Kit-D1; Fuzhou Maixin Biotechnology Development Co., Ltd.) was added and incubated at room temperature for $15 \mathrm{mi}$, following which $100 \mu 1$ freshly prepared 3'3' diaminobenzidine solution was added at room temperature and color developed for 3-10 min. Sections were routinely stained with hematoxylin at room temperature for $1 \mathrm{~min}$, followed by regular dehydration, made transparent and mounted with neutral gum. Microscopic observation was performed with light microscopy at x400 magnification (Eclipse Ci-L; Nikon Corporation).

Image-Pro Plus 6.0 software (Media Cybernetics, Inc.) was used to select the brownish yellow color as a uniform standard for determining the positive staining of all images. Each image was analyzed to determine the average optical density.

Western blotting. Tissue was first lysed using RIPA buffer (cat. no. R0010; Beijing Solarbio Science \& Technology Co., Ltd.). The protein concentration of the mucosal samples was detected by bicinchoninic acid assay (Beijing Solarbio Science $\&$ Technology Co., Ltd.). An equal amount of protein $(40 \mu \mathrm{g})$ of each sample was loaded onto $12 \%$ polyacrylamide gels for electrophoresis and then transferred to a PVDF membrane. After blocking at $25^{\circ} \mathrm{C}$ with $5 \%$ fat-free milk for $1-2 \mathrm{~h}$, the membranes were incubated with anti-claudin-3 antibody (cat. no. ab15102; 1:200; Abcam) and $\beta$-actin (1:5,000; cat. no. 66009-1-lg; ProteinTech Group, Inc.) at $4^{\circ} \mathrm{C}$ overnight. After incubation at $25^{\circ} \mathrm{C}$ with horseradish peroxidase-conjugated secondary antibody (cat. no. SA00001-2, dilution 1:2,000; ProteinTech Group, Inc.) for $2 \mathrm{~h}$, the membranes were visualized by Mini Chemi 610 electrochemiluminescence (Beijing Sage Creation Science Co., Ltd.) and the gray value was analyzed using Lane ID software 5.0 (Beijing Sage Creation Science Co, Ltd.).
Statistical analysis. SPSS 16.0 software (IBM Corp.) was used for statistical analysis. Data are presented as the mean $\pm \mathrm{SD}$. An independent sample t-test was used for comparisons between two groups. Measurement data were analyzed by linear correlation analysis (Pearson's rank correlation test) after the normality test. Bonferroni's correction test was used when analyzing the correlation of multiple groups and the P-value was denoted as $\mathrm{P}$ corrected $(\mathrm{Pc}) . \mathrm{P}<0.05$ was considered to indicate a statistically significant difference.

\section{Results}

APACHE II scores and pathological damage scores. The APACHE score consists of four categories, including APACHE I, II, III and IV, among which APACHE II provides clinical values in prediction of hospital mortality, ICU, length of stay, resource utilization, cost-effectiveness and necessities of receiving positive life support treatment (5); the higher the score, the more severe the illness will be (26). In the present study, the average APACHE II score of the patients was $14.87 \pm 5.6$ points. Furthermore, the average pathological damage score of gastric mucosa was calculated by analyzing the H\&E staining (Fig. 1). The score was $2.92 \pm 1.08$ points in patients with SRMD, which was significantly higher compared with the control group $(1.04 \pm 0.60 ; \mathrm{P}<0.001$; data not shown).

Comparison of serum indexes between patients and volunteers. Serum indexes of oxidative stress in patients with SRMD were analyzed. It was found that the levels of MDA $(4.74 \pm 2.89 \mathrm{nmol} / \mathrm{ml})$ and IMA $(93.61 \pm 10.78 \mathrm{U} / \mathrm{ml})$ in patients with SRMD were significantly higher compared with the healthy controls $(\mathrm{P}<0.001$; Table I). Furthermore, the levels of SOD $(89.66 \pm 12.85 \mathrm{U} / \mathrm{ml})$ in patients with SRMD were significantly lower than compared with the controls $(\mathrm{P}<0.001$; Table I). Thus, these results suggest that the oxidative stress indexes of patients with SRMD were significantly higher, while the antioxidant index was significantly lower.

Comparison of mucosal indexes between patients and volunteers. Next, the mucosal index was compared between patients and healthy controls. More positive staining was identified for iNOS in patients with SRMD compared with healthy controls (Fig. 2). In addition, the mean levels of iNOS in patients were significantly higher compared with the controls (Table II). 
Table I. Comparison of serum index levels between patients and healthy controls.

\begin{tabular}{|c|c|c|c|c|}
\hline Parameter & Patients & Controls & $\mathrm{t}$ & P-value \\
\hline $\operatorname{MDA}(\mathrm{nmol} / \mathrm{ml})$ & $4.74 \pm 2.89$ & $0.55 \pm 0.34$ & 8.769 & $<0.001$ \\
\hline $\mathrm{SOD}(\mathrm{U} / \mathrm{ml})$ & $89.66 \pm 12.85$ & $148.73 \pm 13.50$ & -14.868 & $<0.001$ \\
\hline IMA (U/ml) & $93.61 \pm 10.78$ & $59.07 \pm 7.411$ & 11.358 & $<0.001$ \\
\hline
\end{tabular}

MDA, malondialdehyde; SOD, superoxide dismutase; IMA, ischemic modified albumin.

Table II. Comparison of gastric mucosal index between patients and healthy controls.

\begin{tabular}{lccr}
\hline Protein & Patients & Controls & $\mathrm{t}$ \\
\hline Claudin-3 (gray value) & $0.12 \pm 0.050$ & $0.95 \pm 0.615$ & -5.195 \\
iNOS (average optical density) & $0.1183 \pm 0.0173$ & $0.0578 \pm 0.0031$ & 2.180 \\
\hline
\end{tabular}

iNOS, inducible nitric oxide synthase.
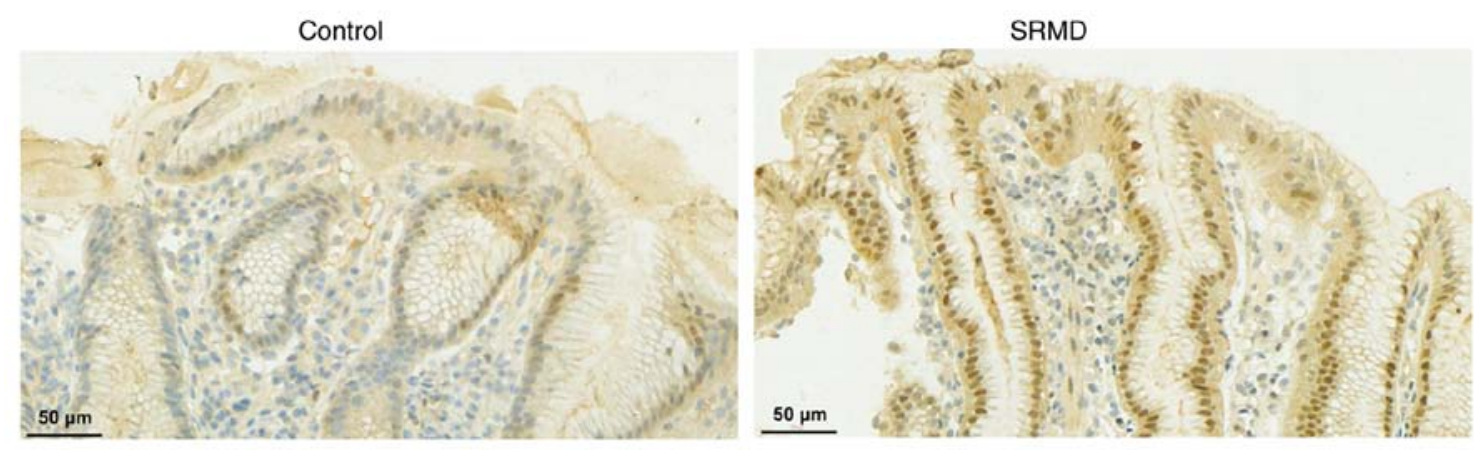

Figure 2. Immunohistochemistry analysis of inducible nitric oxide synthase in mucosal epithelium from healthy control and a patient with SRMD. Magnification x400. Scale bar, $50 \mu \mathrm{m}$. SRMD, stress-related mucosal disease.
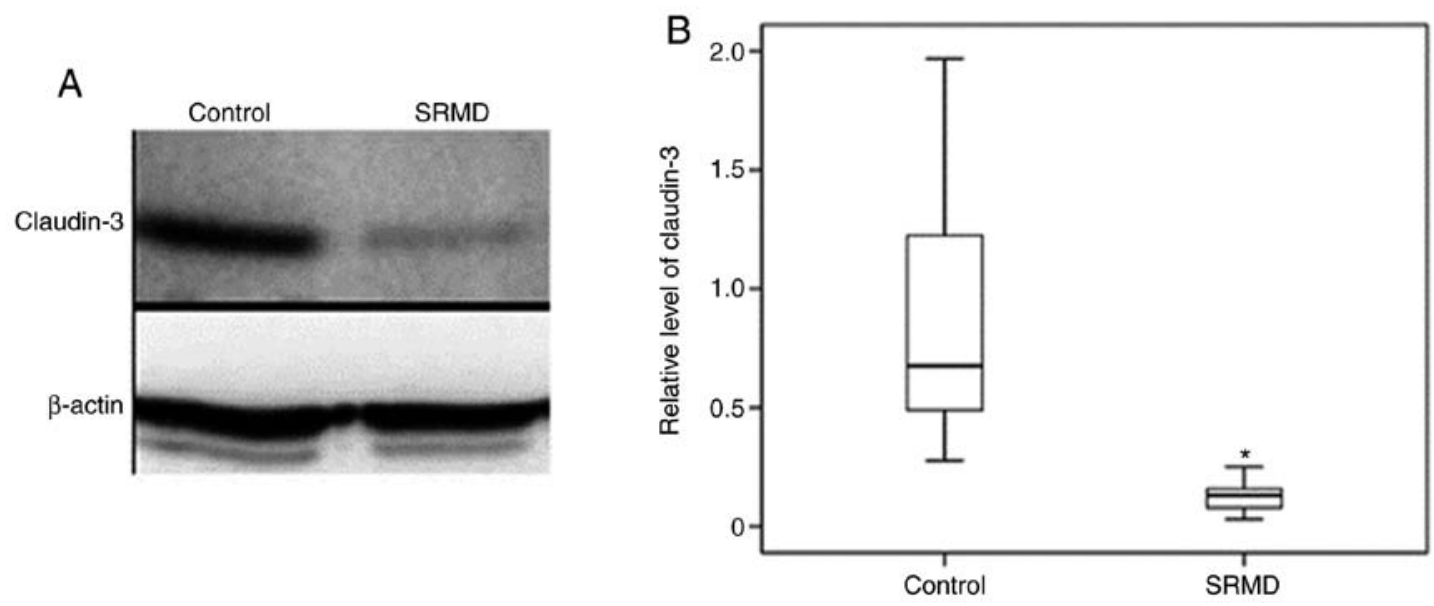

Figure 3. Western blot analysis of claudin-3 in gastric mucosa. (A) Claudin-3 and $\beta$-actin were evaluated by western blotting. (B) Densitometric analysis of claudin- 3 expression normalized to $\beta$-actin. ${ }^{*} \mathrm{P}<0.001$ vs. control. SRMD, stress-related mucosal disease.

Furthermore, claudin-3 expression in mucosal epithelium was measured by western blotting (Fig. 3A), and the mean expression of claudin-3 in patients was significantly lower compared with the controls $(\mathrm{P}<0.001$; Fig. 3B; Table II).
Relationship of APACHE II score with pathological damage score, MDA and SOD. To study the relationship between disease progression and oxidative stress or mucosal damage, correlation analysis was performed. It was demonstrated that 

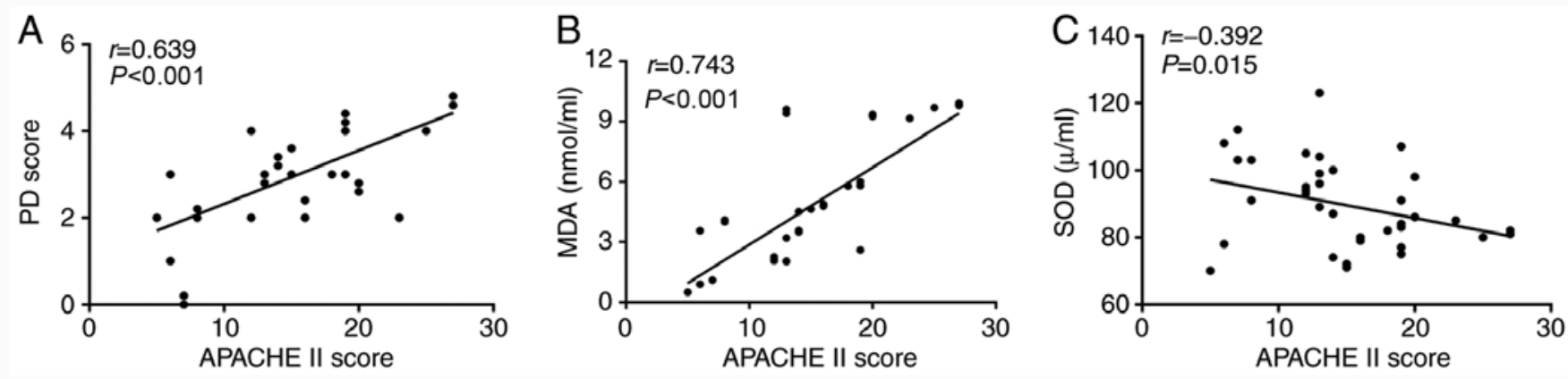

Figure 4. Relationship of APACHE II score with PD score, MDA and SOD. Pearson's rank correlation test was performed for linear correlation analysis. Correlation of APACHE II score with (A) PD score, (B) MDA and (C) SOD. P-value threshold after Bonferroni's correction $<0.016$. PD, pathological damage; SOD, superoxide dismutase; MDA, malondialdehyde; APACHE, Acute Physiology and Chronic Health Evaluation.
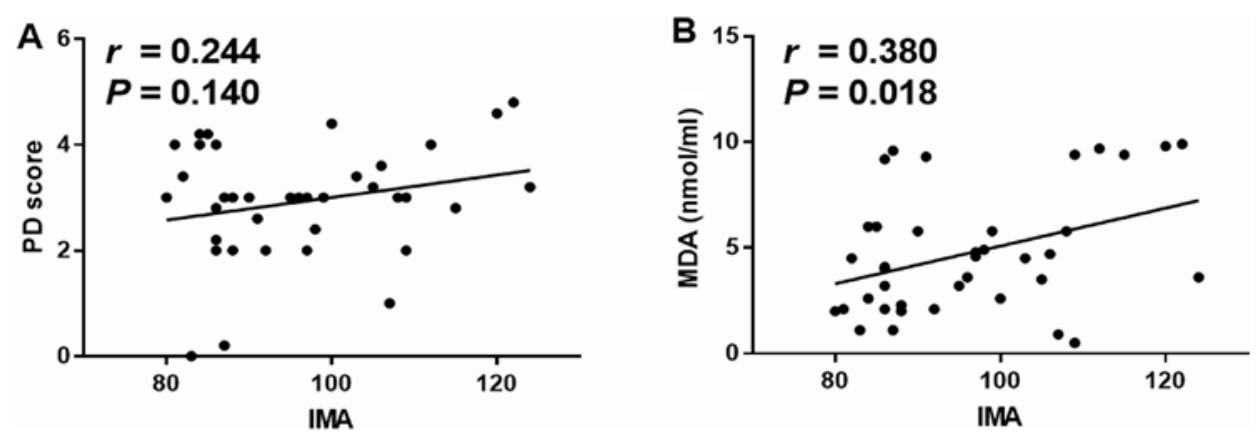

Figure 5. Relationship of IMA with PD score and MDA. Pearson's rank correlation test was performed for linear correlation analysis. Correlation of IMA with (A) PD score and (B) MDA. P-value threshold after Bonferroni's correction <0.025. MDA, malondialdehyde; PD, pathological damage; IMA, ischemic modified albumin.
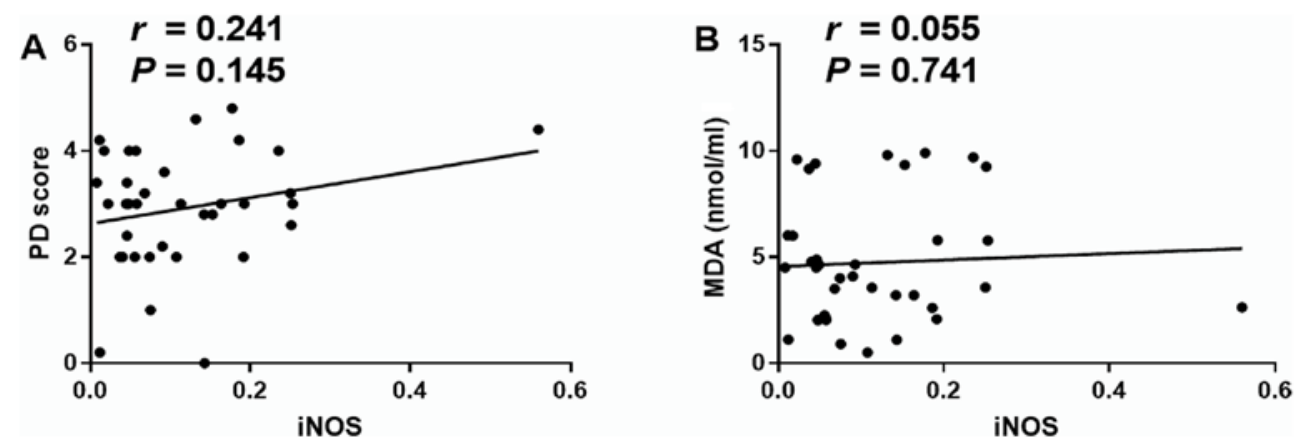

Figure 6. Relationship of iNOS with PD score and MDA. Pearson's rank correlation test was performed for linear correlation analysis. Correlation of iNOS with (A) PD score and (B) MDA. P-value threshold after Bonferroni's correction $<0.025$. PD, pathological damage; MDA, malondialdehyde; iNOS, inducible nitric oxide synthase.

the APACHE II score was moderately positively correlated with pathological damage score $(\mathrm{r}=0.639, \mathrm{P}<0.001)$ and strongly positively correlated with MDA $(r=0.743, \mathrm{P}<0.001$; Fig. 4A and B), and weakly negatively correlated with SOD $(\mathrm{r}=-0.392, \mathrm{P}=0.015$; Fig. 4C; after Bonferroni's correction, $\mathrm{Pc}=0.016$ ). Therefore, it was speculated that oxidative stress was increased with the severity of diseases.

Relationship of IMA with pathological damage score and $M D A$. To evaluate whether IMA can reflect the degree of gastric mucosal lesions, the relationship of IMA with pathological damage score and MDA was analyzed. It was demonstrated that IMA was not significantly correlated with pathological damage score ( $\mathrm{P}=0.140$; Fig. 5A). However, IMA was weakly positively correlated with MDA $(r=0.380, P=0.018$; Fig. 5B; after Bonferroni's correction, $\mathrm{Pc}=0.05 / 2=0.025$ ). Collectively, the results indicated that IMA can reflect the degree of oxidative stress, but not the degree of stress in gastric mucosal lesions.

Relationship of iNOS with pathological damage score and $M D A$. By analyzing the relationship between mucosal iNOS and the mucosal damage or the oxidative stress index, it was indicated that iNOS was not significantly correlated with pathological damage score or MDA ( $\mathrm{P}>0.05$; Fig. 6A and B; after Bonferroni's correction, $\mathrm{Pc}=0.05 / 2=0.025)$. 

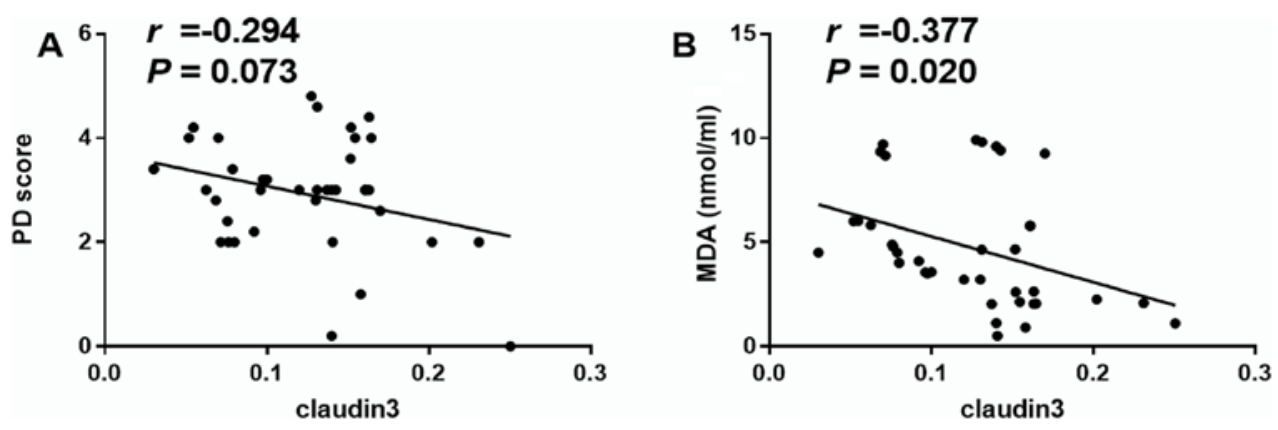

Figure 7. Relationship of claudin-3 with PD score and MDA. Pearson's rank correlation test was performed for linear correlation analysis. Correlation of claudin-3 with (A) PD score and (B) MDA. P-value threshold after Bonferroni's correction <0.025. PD, pathological damage; MDA, malondialdehyde.

Relationship of claudin-3 with pathological damage score and MDA. The relationship between mucosal permeability and mucosal damage or oxidative stress was also investigated. It was identified that claudin-3 was not significantly correlated with pathological damage score $(\mathrm{P}>0.05$; Fig. 7A). However, claudin-3 was weakly negatively correlated with MDA ( $\mathrm{r}=-0.377$; $\mathrm{P}=0.020$; Fig. 7B; after Bonferroni's correction, $\mathrm{Pc}=0.05 / 2=0.025$ ). Thus, it was speculated that mucosal permeability may be related to the degree of oxidative stress.

\section{Discussion}

In clinical practice, SRMD is usually observed in patients in ICU during gastroscopy (1). The present results suggested that the levels of MDA and IMA in the serum of patients with SRMD were significantly higher compared with the healthy controls. However, the level of SOD in the serum of patients with SRMD was significantly lower compared with the controls. Therefore, the results indicated that the antioxidant system could not compete with oxidative stress in patients with SRMD, and thus, it was speculated that oxidative stress may be important in the pathogenesis of SRMD.

MDA is currently the most widely used indicator for the detection of lipid peroxidation products and is often used to reflect the degree of oxidative stress (8). The present results identified significantly elevated MDA levels in patients with SRMD, suggesting that lipid peroxidation induced by ROS may be involved in the formation of gastric mucosal damage in patients with SRMD.

The activity and level of SOD reflects the antioxidant capacity (9). IMA is also an indicator of oxidative stress. Moreover,IMA in the heart and other organs or tissues increases when oxidative stress is induced by ischemia-reperfusion injury $(12,27,28)$. In the hypoxic state, the production of hydroxyl radicals of ROS leads to changes in the $\mathrm{N}$-terminus of albumin and loss of transport metal adhesion, which can produce IMA (29). In the present study, the lower mean value of SOD, as well as higher mean IMA levels in patients with SRMD collectively demonstrated that oxidative stress may be involved in the pathogenesis of SRMD.

Previous studies have reported a correlation between disease severity and oxidative stress state. For example, Lorente et al (30) revealed that the level of MDA in serum is associated with APACHE II score. In addition, Satoh et al (31) showed significantly positive correlations between IMA and APACHE II score. The present results also suggested that APACHE II score was positively correlated with MDA, and negatively correlated with SOD, thus indicating that oxidative stress may be positively correlated with disease severity. Furthermore, there was a positive correlation between APACHE II score and mucosal pathological damage score. Collectively, the results demonstrated that oxidative stress may be an important aspect of the pathogenesis of SRMD.

NO vasodilatation, a basic gastric mucosal defense, provides blood to the mucosa to resist damage from gastric acid and pepsin $(32,33)$. However, the role of NO in the gastrointestinal tract is not just a defense mechanism, and previous studies $(18,19)$ have revealed that NO may play a dual role in ischemia-reperfusion-induced mucosal damage (34). Furthermore, NO is increased in ischemia myocardial tissue following ischemia-reperfusion (35). During ischemic tissue reperfusion, $\mathrm{Ca}^{2+}$ flows into the cells and the formation of oxygen free radicals may lead to ischemia-reperfusion injury (36). Moreover, NO can react with oxygen radicals to yield peroxynitrite, which can cause tissue damage via lipid peroxidation (37). The present results suggested that the expression of iNOS in patients with SRMD was significantly higher compared with healthy volunteers. Therefore, it was speculated that ischemic hypoxic conditions are more severe in patients who are critically ill and that ischemia-reperfusion leads to higher iNOS expression in these patients, which induces the production of large amounts of NO. Thus, under oxidative stress, peroxynitrite produced by the reaction of ROS with NO directly damages tissues and leads to SRMD (17).

Based on the complexity of the pathogenesis of SRMD and the changes of claudin-3 under oxidative stress (23), the present study also investigated the effect of SRMD on claudin-3 expression. It was identified that the expression of claudin-3 in patients with SRMD was significantly lower compared with healthy controls, and claudin-3 expression in patients was negatively correlated with MDA. As a type of ROS, $\mathrm{H}_{2} \mathrm{O}_{2}$ can remain in cells for a long time and act as a second messenger for a variety of physiological stimuli, such as inflammatory cytokines and growth factors (38). It has also been reported that $\mathrm{H}_{2} \mathrm{O}_{2}$ can activate cAMP-dependent protein kinase, which can further phosphorylate claudin-3 $(39,40)$. Thus, it was speculated that $\mathrm{H}_{2} \mathrm{O}_{2}$ phosphorylates claudin- 3 by activating the cAMP-dependent protein kinase, resulting in 
a degradation of claudin-3. Collectively, it was hypothesized that the pathogenesis of SRMD may be associated with the decrease in tight junction proteins and an increase in permeability due to the influence of ROS on claudin-3, but its specific mechanism remains to be elucidated.

However, there were some limitations to the present study. First, the sample size was small. Moreover, the interval between the stress events and the sampling time was different, which may have led to bias. Third, psychological stress may be an important factor affecting the mucosal barrier in patients who are critically ill (41), but this factor was not included in this study due to practical difficulties.

In conclusion, the present results suggested that there was oxidative stress in patients who are critically ill with SRMD, and that the degree of oxidative stress may be related to disease severity of patients in ICU. Therefore, oxidative stress may be an important aspect of the pathogenesis of SRMD. However, oxidative stress may lead to SRMD via increased NO. Moreover, oxidative stress may increase the permeability of cell membrane by affecting claudin-3 expression. Thus, future studies should focus on factors related to oxidative stress to identify sensitive indicators of SRMD and to avoid over-medication for patients who are low-risk.

\section{Acknowledgements}

Not applicable.

\section{Funding}

No funding was received.

\section{Availability of data and materials}

All data generated or analyzed during this study are included in this published article.

\section{Authors' contributions}

XW and XZ designed the study. XW, QZ, HS, FQ, NS, DB and $\mathrm{HY}$ contributed to the data acquisition and conducted the experiments. QZ, HS and XL performed the statistical analysis. XW and DB prepared the manuscript. QZ, HS and NS conducted the literature search. All authors read and approved the final version of the manuscript.

\section{Ethics approval and consent to participate}

This study was approved by the Institutional Ethics Committee of Tai'an Central Hospital. All subjects provided their informed consent. The study was registered at www.clinicaltrials.gov (study ID. NCT03200158).

\section{Patient consent for publication}

Not applicable.

\section{Competing interests}

The authors declare that they have no competing interests.

\section{References}

1. Kantorova I, Svoboda P, Scheer P, Doubek J, Rehorkova D Bosakova $\mathrm{H}$ and Ochmann J: Stress ulcer prophylaxis in critically ill patients: A randomized controlled trial. Hepatogastroenterology 51: 757-761, 2004.

2. Lin PC, Chang CH, Hsu PI, Tseng PL and Huang YB: The efficacy and safety of proton pump inhibitors vs. histamine-2 receptor antagonists for stress ulcer bleeding prophylaxis among critical care patients: A meta-analysis. Crit Care Med 38: 1197-1205, 2010.

3. Chu YF, Jiang Y, Meng M, Jiang JJ, Zhang JC, Ren HS and Wang CT: Incidence and risk factors of gastrointestinal bleeding in mechanically ventilated patients. World J Emerg Med 1: 32-36, 2010.

4. Knaus WA, Draper EA, Wagner DP and Zimmerman JE: APACHE II: A severity of disease classification system. Crit Care Med 13: 818-829, 1985.

5. Niewiński G, Starczewska M and Kański A: Prognostic scoring systems for mortality in intensive care units-the APACHE model. Anaesthesiol Intensive Ther 46: 46-49, 2014.

6. Ali T and Harty RF: Stress-induced ulcer bleeding in critically ill patients. Gastroenterol Clin North Am 38: 245-265, 2009.

7. Preiser JC: Oxidative stress. JPEN J Parenter Enteral Nutr 36: 147-154, 2012.

8. Spirlandeli AL, Deminice R and Jordao AA: Plasma malondialdehyde as biomarker of lipid peroxidation: Effects of acute exercise. Int J Sports Med 35: 14-18, 2014.

9. Kwiecień S, Brzozowski T and Konturek SJ: Importance of aldehyde products of lipid peroxidation in the formation of gastric lesions induced by aspirin, ischemia-reperfusion and stress. Gastroenterol Polska 9: 273-280, 2002.

10. Matsuda T, Tao H, Goto M, Yamada H, Suzuki M, Wu Y, Xiao N, He Q, Guo W, Cai Z, et al: Lipid peroxidation-induced DNA adducts in human gastric mucosa. Carcinogenesis 34: 121-127, 2013.

11. Kwiecień S, Pawlik MW, Brzozowski T, Pawlik WW and Konturek SJ: Reactive oxygen metabolite action in experimental, stress model of gastric mucosa damage. Gastroenterol Polska 17: 234-243, 2010.

12. Borderie D, Allanore Y, Meune C, Devaux JY, Ekindjian OG and Kahan A: High ischemiamodifed albumin concentration reflects oxidative stress but not myocardial involvement in systemic sclerosis. Clin Chem 50: 2190-2193, 2004.

13. Aydin O, Ellidag HY, Eren E, Kurtulus F, Yaman A and Yilmaz N: Ischemia modified albumin is an indicator of oxidative stress in multiple sclerosis. Biochem Med (Zagreb) 24: 383-389, 2014.

14. Gothe PR, Jose M, Pai VR, Harish S, D'Souza J and Prabhu V: Investigation of the possibility of using serum ischemia modified albumin (IMA) as a novel and early marker of the extent of oxidative stress induced by various tobacco products. J Clin Diagn Res 9: ZC33-ZC35, 2015.

15. Martin MJ, Jimenez MD and Motilva V: New issues about nitric oxide and its effects on the gastrointestinal tract. Curr Pharm Des 7: 881-908, 2001.

16. Stanek A, Gadowska-Cicha A, Gawron K, Wielkoszyński T, Adamek B, Cieślar G, Wiczkowski A and Sieroń A: Role of nitric oxide in physiology and pathology of the gastrointestinal tract. Mini Rev Med Chem 8: 1549-1560, 2008.

17. Kobata A, Kotani T, Komatsu Y, Amagase K, Kato S and Takeuchi K: Dual action of nitric oxide in the pathogenesis of ischemia/reperfusion-induced mucosal injury in mouse stomach. Digestion 75: 188-197, 2007.

18. Tanaka A,Kunikata T, Mizoguchi H, Kato S and Takeuchi K: Dual action of nitric oxide in pathogenesis of indomethacin-induced small intestinal ulceration in rats. J Physiol Pharmacol 50: 405-417, 1999.

19. Whittle BJ, Laszlo F, Evans SM and Moncada S: Induction of nitric oxide synthase and microvascular injury in the rat jejunum provoked by indomethacin. Br J Pharmacol 116: 2286-2290, 1995.

20. Wu J, Yang Y, Xun N, Zeng L, Li Z, Yang W, Liang Y, Ma Z and Tang H: Osthole attenuates myocardial ischemia/reperfusion injury in rats by inhibiting apoptosis and inflammation. Am J Transl Res 10: 1109-1116, 2018.

21. Zhu L, Wei T, Gao J, Chang X, He H, Luo F, Zhou R, Ma C, Liu Y and Yan T: The cardioprotective effect of salidroside against myocardial ischemia reperfusion injury in rats by inhibiting apoptosis and inflammation. Apoptosis 20: 1433-1443, 2015. 
22. Patel RM, Myers LS, Kurundkar AR, Maheshwari A, Nusrat A and Lin PW: Probiotic bacteria induce maturation of intestinal claudin 3 expression and barrier function. Am J Pathol 180: 626-635, 2012.

23. Hashimoto K, Oshima T, Tomita T, Kim Y, Matsumoto T, Joh T and Miwa $\mathrm{H}$ : Oxidative stress induces gastric epithelial permeability through claudin-3. Biochem Biophys Res Commun 376: 154-157, 2008.

24. Masuda E, Kawano S, Nagano K, Tsuji S, Takei Y, Hayashi N, Tsujii M, Oshita M, Michida T and Kobayashi I: Role of endogenous endothelin in pathogenesis of ethanol-induced gastric mucosal injury in rats. Am J Physiol 265: G474-G481, 1993.

25. Yagi K: A simple fluorometric assay for lipoperoxide in blood plasma. Biochem Med 15: 212-216, 1976.

26. Swann JW, Maunder CL, Roberts E, McLauchlan G and Adamantos S: Prevalence and risk factors for development of hemorrhagic gastro-intestinal disease in veterinary intensive care units in the United Kingdom. J Vet Emerg Crit Care (San Antonio) 26: 419-427, 2016.

27. Sbarouni E, Georgiadou P and Voudris V: Ischemia modified albumin changes-review and clinical implications. Clin Chem Lab Med 49: 177-184, 2011

28. Sharma R, Gaze DC, Pellerin D, Mehta RL, Gregson H, Streather CP, Collinson PO and Brecker SJ: Ischemia-modified albumin predicts mortality in ESRD. Am J Kidney Dis 47 493-502, 2006

29. Apple FS, Quist HE, Otto AP, Mathews WE and Murakami MM: Release characteristics of cardiac biomarkers and ischemiamodified albumin as measured by the albumin cobalt-binding test after a marathon race. Clin Chem 48: 1097-1100, 2002.

30. Lorente L, Martín MM, Abreu-Gonzalez P, DomínguezRodríguez A, Labarta L, Díaz C, Solé-Violán J, Ferreres J, Borreguero-León JM, Jiménez A and Morera-Fumero A: Prognostic value of malondialdehyde serum levels in severe sepsis: A multicenter study. PLoS One 8: e53741, 2013.

31. Satoh M, Kotani K, Gugliucci A, Horie H, Caccavello R and Takeuchi M: Correlation of ischemia-modified albumin with SOFA and APACHE II scores in preoperative patients with colorectal cancer. ScientificWorld Journal 2014: 959075, 2014.

32. Kotani T, Kobata A, Nakamura E, Amagase K and Takeuchi K: Roles of cyclooxygenase- 2 and prostacyclin/IP receptors in mucosal defense against ischemia/reperfusion injury in mouse stomach. J Pharmacol Exp Ther 316: 547-555, 2006.
33. Naito Y, Yoshikawa T, Matsuyama K, Yagi N, Arai M, Nakamura Y, Kaneko T, Yoshida N and Kondo M: Neutrophils, lipid peroxidation, and nitric oxide in gastric reperfusion injury in rats. Free Radic Biol Med 24: 494-502, 1998.

34. Lopez-Belmonte J, Whittle BJ and Moncada S: The actions of nitric oxide donors in the prevention or induction of injury to the rat gastric mucosa. Br J Pharmacol 108: 73-78, 1993.

35. Zhu T, Yao Q, Wang W, Yao $\mathrm{H}$ and Chao J: INOS induces vascular endothelial cell migration and apoptosis via autophagy in ischemia/reperfusion injury. Cell Physiol Biochem 38: $1575-1588,2016$

36. Okuda M, Lee HC, Chance B and Kumar C: Role of extracellular $\mathrm{Ca} 2+$ in ischemia-reperfusion injury in the isolated perfused rat liver. Circ Shock 37: 209-219, 1992.

37. Dijkstra G, Moshage H, van Dullemen HM, de Jager-Krikken A, Tiebosch AT, Kleibeuker JH, Jansen PL and van Goor H: Expression of nitric oxide synthases and formation of nitrotyrosine and reactive oxygen species in inflammatory bowel disease. J Pathol 186: 416-421, 1998.

38. Bae YS, Kang SW, Seo MS, Baines IC, Tekle E, Chock PB and Rhee SG: Epidermal growth factor (EGF)-induced generation of hydrogen peroxide. Role in EGF receptor-mediated tyrosine phosphorylation. J Biol Chem 272: 217-221, 1997.

39. Bai XC, Lu D, Liu AL, Zhang ZM, Li XM, Zou ZP, Zeng WS, Cheng BL and Luo SQ: Reactive oxygen species stimulates receptor activator of NF-kappaB ligand expression in osteoblast. J Biol Chem 280: 17497-17506, 2005.

40. D'Souza T, Agarwal R and Morin PJ: Phosphorylation of claudin-3 at threonine 192 by cAMP-dependent protein kinase regulates tight junction barrier function in ovarian cancer cells. J Biol Chem 280: 26233-26240, 2005.

41. De R, Mazumder S, Sarkar S, Debsharma S, Siddiqui AA, Saha SJ, Banerjee C, Nag S, Saha D and Bandyopadhyay U: Acute mental stress induces mitochondrial bioenergetic crisis and hyper-fission along with aberrant mitophagy in the gut mucosa in rodent model of stress-related mucosal disease. Free Radic Biol Med 113: 424-438, 2017.

This work is licensed under a Creative Commons Attribution-NonCommercial-NoDerivatives 4.0 International (CC BY-NC-ND 4.0) License. 